\title{
Rational and combinatorial approaches to engineering styrene production by Saccharomyces cerevisiae
}

\author{
Rebekah McKenna, Brian Thompson, Shawn Pugh and David R Nielsen*
}

\begin{abstract}
Background: Styrene is an important building-block petrochemical and monomer used to produce numerous plastics. Whereas styrene bioproduction by Escherichia coli was previously reported, the long-term potential of this approach will ultimately rely on the use of hosts with improved industrial phenotypes, such as the yeast Saccharomyces cerevisiae.

Results: Classical metabolic evolution was first applied to isolate a mutant capable of phenylalanine overproduction to $357 \mathrm{mg} / \mathrm{L}$. Transcription analysis revealed up-regulation of several phenylalanine biosynthesis pathway genes including $A R O 3$, encoding the bottleneck enzyme DAHP synthase. To catalyze the first pathway step, phenylalanine ammonia lyase encoded by PAL2 from A. thaliana was constitutively expressed from a high copy plasmid. The final pathway step, phenylacrylate decarboxylase, was catalyzed by the native FDC1. Expression of FDC1 was naturally induced by trans-cinnamate, the pathway intermediate and its substrate, at levels sufficient for ensuring flux through the pathway. Deletion of $A R O 10$ to eliminate the competing Ehrlich pathway and expression of a feedback-resistant DAHP synthase encoded by $A R O 4^{K 229 L}$ preserved and promoted the endogenous availability precursor phenylalanine, leading to improved pathway flux and styrene production. These systematic improvements allowed styrene titers to ultimately reach $29 \mathrm{mg} / \mathrm{L}$ at a glucose yield of $1.44 \mathrm{mg} / \mathrm{g}$, a $60 \%$ improvement over the initial strain.
\end{abstract}

Conclusions: The potential of S. cerevisiae as a host for renewable styrene production has been demonstrated. Significant strain improvements, however, will ultimately be needed to achieve economical production levels.

Keywords: Styrene, Yeast, Phenylalanine, Aromatics

\section{Background}

Similar to most other monomers used in conventional plastics production, at present, styrene is derived exclusively from non-renewable feedstocks. More specifically, current styrene production predominantly involves the energy-intensive chemocatalytic dehydrogenation of petroleum-derived ethylbenzene [1,2]. With the global annual demand of styrene expected to surpass 41 million tons by 2020 [3], representing a > $\$ 28$ billion U.S. market [4], net energy requirements associated with just this single conversion will amount to over 200 trillion BTU of steam each year [5]. Accordingly, concerns over

\footnotetext{
* Correspondence: David.R.Nielsen@asu.edu

Chemical Engineering, School for Engineering of Matter, Transport, and Energy, Arizona State University, PO Box 876106, Tempe, AZ 85287-6106, USA
}

depleting feedstock availability and deleterious environmental impacts, continue to drive interest in developing 'green' processes for producing biorenewable replacements to conventional petrochemicals, including monomers such as styrene.

Advances in metabolic and pathway engineering continue to expand the range of conventional monomer compounds that can be synthesized from renewable biomass feedstocks [6-9]. Along these lines, the engineering of a novel and non-natural pathway for styrene biosynthesis from biomass-derived glucose was recently reported using the bacterium Escherichia coli as host [10]. Said pathway, which is illustrated in Figure 1, utilizes endogenous phenylalanine as its immediate precursor. Phenylalanine is first deaminated to trans-cinnamate by phenylalanine ammonia lyase (PAL), encoded by PAL2 


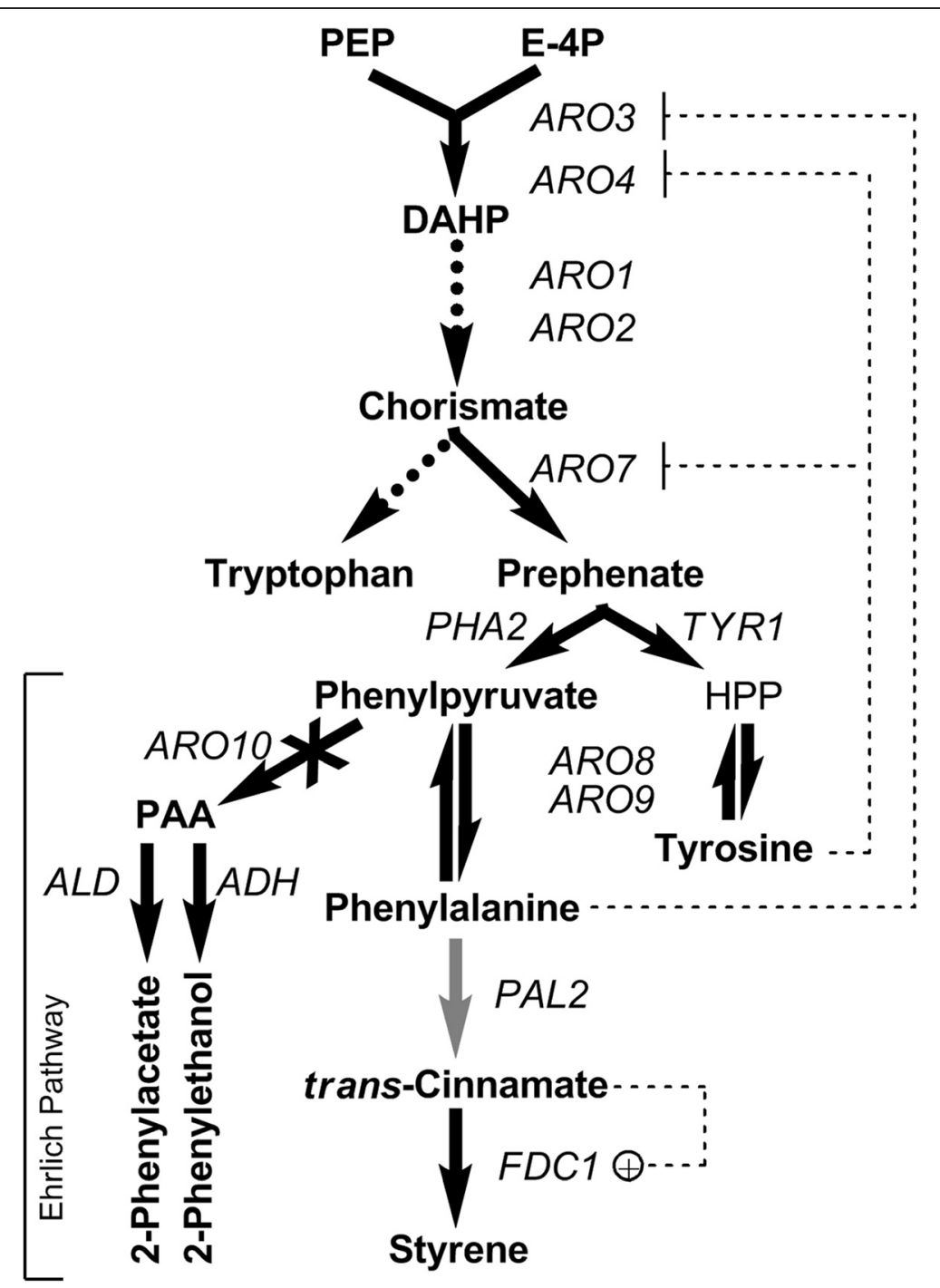

Figure 1 Styrene biosynthesis by engineered S. cerevisiae. Dashed arrows signify multiple steps are involved but not illustrated. Black arrows represent enzymes steps native to S. cerevisiae whereas gray arrows are heterologous; dotted arrows represent multiple enzymatic steps; feedback repression is shown using thin dotted lines with flat heads whereas transcriptional activation is shown using thin dotted lines with a round head and ' + '; disruption of a gene or regulatory mechanism is signified by ' $X$ '. Abbreviations: phosphoenolpyruvate (PEP), erythrose-4phosphate (E-4P), 3-deoxy-D-arabino-heptulosonate-7-phosphate (DAHP), hydroxyphenylpyruvate (HPP), 2-phenylacetaldehyde (PAA).

from Arabidopsis thaliana. Next, trans-cinnamate is decarboxylated to styrene via phenylacrylate decarboxylase, encoded by FDC1 from Saccharomyces cerevisiae $[10,11]$. Co-expressing PAL2 and FDC1 in a previouslyengineered phenylalanine over-producing $E$. coli background resulted in styrene titers as high as $\sim 260 \mathrm{mg} / \mathrm{L}$ (2.5 mM) in glucose minimal media after $29 \mathrm{~h}$, representing a glucose yield of $\sim 0.07 \mathrm{~g} / \mathrm{g}(0.12 \mathrm{~mol} / \mathrm{mol} ; 25 \%$ of theoretical) [10]. As a commodity chemical, however, said production metrics must be improved for biologically derived styrene to emerge as a viable alternative to its conventional counterpart [12].

The engineering of more robust hosts for renewable chemical production is an important aim in industrial biotechnology [13] and, relative to $E$. coli, yeast biosynthetic platforms often afford several inherent attributes of importance to robust and large-scale renewable chemicals production. Said phenotypes most significantly include faster growth rates, elevated solvent tolerance, and the ability to withstand low temperatures and $\mathrm{pH}$ [14-18]. Amongst yeast, S. cerevisiae is a particularly attractive host for metabolic engineering efforts owing to its well characterized genetics, physiology, and metabolism, as well as due to the availability of diverse genetic toolkits for its engineering [19]. Past studies have demonstrated that $S$. cerevisiae is a suitable host for the renewable production of useful aromatic compounds, including protocatechuate, catechol, vanillin, naringenin, 
and 2-phenylethanol [20-23], production of styrene, however, has not yet been reported.

The objective of the present study was to demonstrate that styrene biosynthesis from glucose could be systematically engineered in $S$. cerevisiae. In addition to the aforementioned phenotypic advantages afforded by the use of S. cerevisiae, this aim was further motivated by other relevant factors. For instance, it was hypothesized that improved function of styrene pathway enzymes might be realized in $S$. cerevisiae since: i) PAL2 is of eukaryotic origin, and ii) FDC1 is native to $S$. cerevisiae. Furthermore, contingent upon the native regulation of FDC1 expression (as investigated in this study), it is plausible that a functional styrene pathway could be constructed by expressing a single heterologous enzyme (i.e., PAL2), thereby minimizing the effects of metabolic burden. Meanwhile, as $S$. cerevisiae lacks a natural transporter for phenylalanine efflux [24], it was hypothesized that increased intracellular retention of phenylalanine might enhance its availability to the engineered pathway. These unique features position $S$. cerevisiae as particularly promising host for renewable styrene production. In this study, classical anti-metabolite selection was first applied to evolve a $S$. cerevisiae strain capable of overproducing phenylalanine, the styrene pathway precursor. Rational genetic engineering approaches were used to construct the non-natural styrene pathway and further boost precursor availability. While not before applied for styrene bioproduction, this basic approach has been proven effective for engineering bacterial producers of other aromatic chemicals [25-28].

\section{Results and discussion}

Evolving phenylalanine over-production by S. cerevisiae

As phenylalanine serves as the immediate endogenous precursor to the styrene pathway, its over-production by $S$. cerevisiae is an essential pre-requisite to styrene biosynthesis. Thus, to develop a phenylalanine overproduction phenotype in $S$. cerevisiae, a classic antimetabolite selection strategy was first employed [29]. In this case, $m$-fluoro-DL-phenylalanine was chosen to provide the necessary selection pressure whereas exposure to the chemomutagen EMS increased mutation rates and frequency [30]. In the first round of selection, a total of only two mutants were isolated when using either $18 \mathrm{mg} / \mathrm{L}$ (strain 18A) or $22 \mathrm{mg} / \mathrm{L}$ (strain 22A) $m$-fluoroDL-phenylalanine. As seen in Figure 2, said mutants were subsequently characterized in shake flask cultures. Since phenylalanine is not exported from S. cerevisiae, the established practice of correlating enhanced flux through the pathway with net extracellular accumulation of 2-phenylethanol and 2-phenylacetate (both of which are naturally and readily produced as degradation products of phenylpyruvate, the precursor to phenylalanine; Figure 1) was employed [24]. In S. cerevisiae, it has previously been shown that all available phenylalanine is efficiently shuttled through one or both of these pathways [31], with the relative distribution of products being

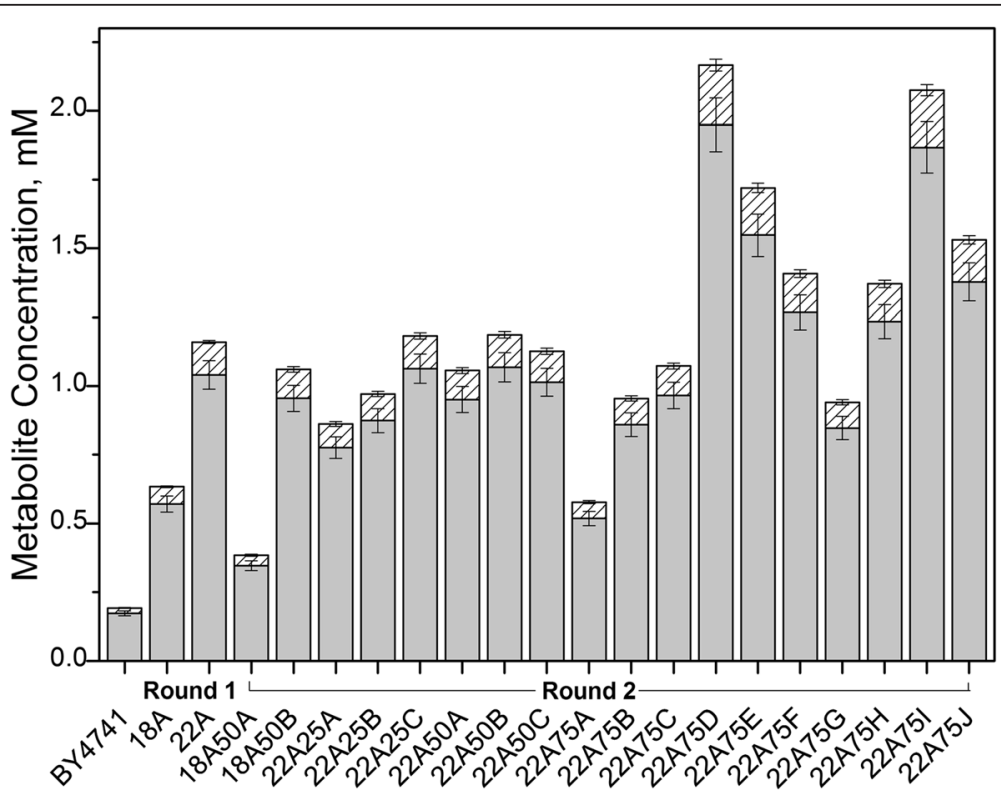

Figure 2 Evolution of phenylalanine overproducing mutants of S. cerevisiae. Mutants were evolved through the use of EMS mutagenesis and high-throughput selection on solid agar plates using $m$-fluoro-DL-phenylalanine as anti-metabolite. 2-Phenylethanol (gray) and 2phenylacetate (hashed) production by isolated mutants was determined after $48 \mathrm{~h}$ of growth in SD media by measuring the concentration of 2phenylethanol and 2-phenylacetate in the supernatant. Error bars reported at one standard deviation from triplicate experiments. 
dictated by the cell's redox state (specifically, the relative intracellular ratio of $\mathrm{NAD}+$ to $\mathrm{NADH}$ ) [32]. For example, in glucose grown cultures with limited aeration (as would be expected in the sealed shake flasks used in this study), 9:1 mixtures of 2-phenylethanol:2-phenylacetate are typically observed [32]. Throughout this study, the mixture of products obtained was similarly consistent (an average of 89\% 2-phenylethanol; Figure 2). Relative to BY4741 (the parent strain and control), strains 18A and 22A showed 3.3- and 6.4-fold improvements in net production of 2-phenylethanol and 2-phenylacetate $(0.63 \pm 0.05$ and $1.13 \pm 0.10 \mathrm{mM})$, respectively.

To further deregulate and enhance phenylalanine biosynthesis, a second round of mutagenesis and selection was performed, in this case using the isolated mutants $18 \mathrm{~A}$ and $22 \mathrm{~A}$ as parents. The selection pressure was accordingly elevated by increasing the content of $m$ fluoro-DL-phenylalanine in SD agar plates to $25 \mathrm{mg} / \mathrm{L}$, $50 \mathrm{mg} / \mathrm{L}$, or $75 \mathrm{mg} / \mathrm{L}$. In contrast to the first round, in this case numerous colonies (i.e., > 50) were obtained at all three selection pressures. Thus, to screen just for the best performers, only the fastest growing mutants were selected (i.e., those whose colony forming units were largest after overnight incubation). A total of 18 additional mutants were chosen and subsequently characterized, as above. As seen in Figure 2, the top performing mutant, 22A75D, produced a combined total of about $2.17 \pm$ $0.22 \mathrm{mM}$ 2-phenylethanol and 2-phenylacetate in $48 \mathrm{~h}$, representing $\sim 3.3$ - and $\sim 21$-fold improvements over $22 \mathrm{~A}$ and BY4741, respectively.

\section{Investigating the evolved phenotypes}

A series of characterizations were next performed on strains 22A and 22A75D (with BY4741 as control) to begin to understand the underlying factors responsible for imparting the evolved phenotypes. The native aromatic amino acid biosynthesis pathways of $S$. cerevisiae are shown in Figure 1, where it can be seen that two known control points are principally responsible regulating metabolite flux. The first occurs at DAHP synthase (for which $S$. cerevisiae possesses two isoenzymes), which is allosterically feedback inhibited by either phenylalanine (ARO3) or tyrosine (ARO4) [33-35]. The second, meanwhile, occurs at chorismate mutase (ARO7), which converts chorismate to prephenate, the precursor to both phenylalanine and tyrosine. Transcription of ARO7 is repressed in the presence of as little as $0.5 \mathrm{mM}$ tyrosine but remains, however, insensitive to phenylalanine $[33,36,37]$. Here, overcoming feedback repression of ARO3 thus constitutes a key priority. However, whereas relief from tyrosine repression of ARO4 has been reported to result from a single mutation (K229L) [24], a phenylalanine feedback resistant mutant of ARO3 remains unreported to date.
Sequences of several key genes in the phenylalanine biosynthesis pathway (ARO3, ARO4, ARO7, ARO8, and $P H A 2)$ were first determined for all three strains (including coding regions as well as 500 bp upstream of each start codon). Interestingly, however, mutations were not observed in the sequence of any investigated gene, including with respect to both its coding and upstream non-coding regions. Transcription levels of all genes in the phenylalanine biosynthesis pathway (namely $A R O 1$, ARO2, ARO3, ARO4, ARO7, ARO8, ARO9, and PHA2; see Figure 1) were next examined in the mutants $22 \mathrm{~A}$ and $22 \mathrm{~A} 75 \mathrm{D}$ and quantified relative to that of the wildtype control (BY4741). The results are compared in Figure 3, wherein it can be seen that, in strain 22A75D, up-regulation of $A R O 8$ was found to be most significant (a 9.3-fold increase), followed by ARO1 (6.8-fold), ARO2 (5.8-fold), and ARO3 (4.5-fold). Note that similar but less significant differences were also observed in strain 22A. Furthermore, only modest increases in ARO4 and ARO7 expression were observed in 22A75D (about 2.7and 1.8-fold, respectively), with no significant changes occurring in 22A for either gene.

The collective findings point to the prospects of several interesting mechanisms in the mutant strains. For example, with no change to its sequence, the evolved phenotype clearly did not arise as a result of relieving allosteric inhibition at the known bottleneck enzyme, ARO3. However, as a significant increase in its expression was observed in both mutants, this could suggest that up-regulation of wild type ARO3 occurred as an alternative strategy. That is, despite the fact that the wild type enzyme possesses lower specific activity in the presence of phenylalanine, with more copies net DAHP synthase activity may have been sufficiently high so as to effectively overcome the flux bottleneck. Simultaneous up-regulation of $A R O 1$ and ARO2 (the two subsequent steps in the pathway), meanwhile, may have aided in this process by ensuring that produced DAHP was then promptly assimilated further along the pathway, thereby maintaining a maximum driving force. Up-regulated expression of $A R O 1$ has been successfully employed in yeast as a rational approach for enhancing the biosynthesis of cis,cis-muconic acid - a product derived from the shikimate pathway intermediate 3-hydroshikimate [38]. Meanwhile, up-regulation of ARO8 was evolved perhaps as a mechanism to compete with the native activity of ARO10, ensuring that metabolite flux was efficiently routed towards phenylalanine biosynthesis rather than through the degradative Ehrlich pathway [32,39]. This prospect is further supported by the fact that, in contrast to $A R O 8$, no appreciable change was observed with respect to the expression of $A R O 9$, which functions primarily in the reverse direction (i.e., for phenylalanine assimilation from the culture medium) [40]. 


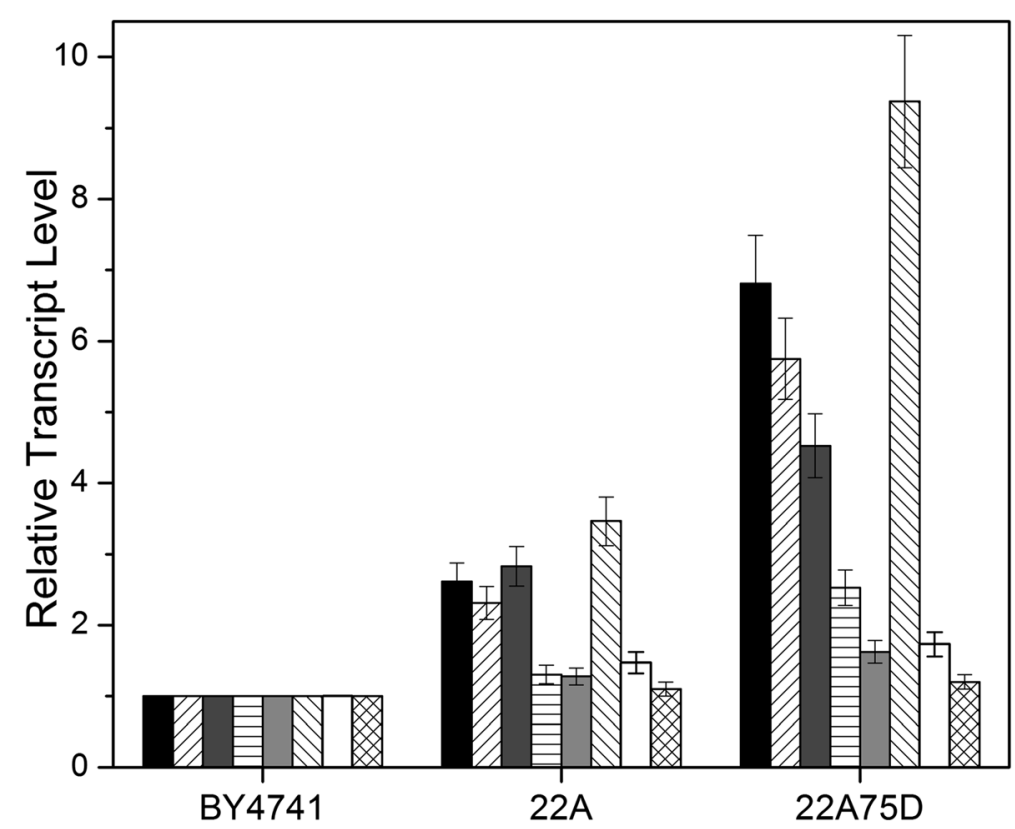

Figure 3 Transcriptional analysis of top phenylalanine overproducing S. cerevisiae mutants. Relative transcript levels of the top first (22A) and second (22A75D) round evolved yeast mutants, normalized to the parent (BY4741). Measured genes included ARO1 (black), ARO2 (right diagonal), ARO3 (dark gray), ARO4 (horizontal), ARO7 (light gray), ARO8 (left diagonal), ARO9 (no fill), and PHA2 (hashed). Error bars reported at one standard deviation from triplicate experiments.

With no change to either the coding or upstream noncoding regions for any of the four up-regulated genes (ARO1, ARO2, ARO3, and ARO8) another factor must be responsible for this observed result. Increased copy number through genomic amplifications is one possibility, however, a more efficient mechanism may have involved the mutation of one or more transcription factors controlling their expression. GCN4 encodes one such major transcription factor [41], however, further sequencing revealed no changes there either. To identify other prospective transcription factors involved, the promoter regions of the four up-regulated genes were further investigated by aligning the $1000 \mathrm{bp}$ sequences prior to each start codon. Whereas a possible consensus sequence of 5'-AACATC-3' was located at positions -292, -307, -289, and -290 for ARO1, ARO2, ARO3, and $A R O 8$, respectively, said sequence does not match binding site of any known transcription factor. Among all known transcriptional regulators, eleven are shared between the four up-regulated genes [42] (note, an annotated list is provided in Additional file 1: Table S3). The ability to determine which if any of these regulators are responsible for the evolved changes will only be possible through the collective analysis of their gene sequences, or better, to ensure full elucidation of all changes in the mutants, through whole-genome sequencing. However, given that the achievable titers are still quite modest (a total of only $2.17 \pm 0.22 \mathrm{mM} 2$-phenylethanol and 2- phenylacetate), such an undertaking was deemed as unwarranted at this time. For now, and for the purpose of this study, these efforts were successful in developing a host strain to serve as a test platform for engineering styrene biosynthesis from glucose.

\section{Investigating native FDC1 activity and factors influencing its expression}

Although it had previously been shown that, when cultured in the presence of exogenous trans-cinnamate, $S$. cerevisiae is capable of catalyzing its decarboxylation to styrene [11], several factors related to the native function and expression of FDC1 remained initially unclear and deserving of further investigation. Most importantly, it was wholly unknown as to if, when, and how the native expression of FDC1 would be induced in the context of the styrene pathway and under the culture conditions of interest. BY4741 was initially cultured in SD minimal media supplemented with potential inducers of interest. In addition to trans-cinnamate and phenylalanine (the pathway intermediate and precursor, respectively), $p$ coumarate and ferulate were also screened as positive controls (note, both are structural homologs of transcinnamate and known inducers of trans-cinnamate decarboxylase activity [11]). As seen in Table 1, in vitro trans-cinnamate decarboxylase activity was detected in the lysates of cells cultured in the presence of each of trans-cinnamate, $p$-coumarate, and ferulate (with the 
Table 1 Assaying the in vitro decarboxylase activity of FDC1 against a pool of structurally-related, phenylacrylic acid substrates

\begin{tabular}{|c|c|c|}
\hline Compound & Induced activity & $\mathrm{mU} \mathrm{mg}^{-1}$ total protein \\
\hline trans-cinnamate & + & $0.46 \pm 0.02$ \\
\hline p-coumarate & + & $0.39 \pm 0.02$ \\
\hline ferulate & + & $0.21 \pm 0.03$ \\
\hline phenylalanine & - & N.D. \\
\hline none (control) & - & N.D. \\
\hline
\end{tabular}

Positive, '+'; Negative, '-'; Not Detected, 'N.D.'.

former serving as the strongest inducer), but not with phenylalanine or in the control. With respect to the styrene pathway, this implies that native FDC1 expression will be wholly contingent upon the heterologous expression of PAL2 to provide trans-cinnamate as inducer (a realization that could be of benefit with respect to minimizing overall metabolic burden).

\section{Evaluating the styrene pathway via the exogenous addition of phenylalanine}

Preliminary studies were next performed to begin probing the functionality of the styrene pathway in wild type $S$. cerevisiae. Strains BY4741-PAL and BY4741 $F$ FDC1-PAL were first cultured in SD-Leu minimal media supplemented with $200 \mathrm{mg} / \mathrm{L}(1.21 \mathrm{mM})$ phenylalanine while monitoring the extracellular accumulation of trans-cinnamate, styrene, and 2-phenylethanol, 2-phenylacetate, and concomitant depletion of phenylalanine. As seen in Figure 4, while only 37\% of supplied the phenylalanine was consumed by BY4741PAL after $24 \mathrm{~h}$, styrene and 2-phenylethanol constituted the major end-products, reaching titers of up to $20 \pm 1$ and $43 \pm 1 \mathrm{mg} / \mathrm{L}(0.19 \pm 0.01$ and $0.35 \pm 0.01 \mathrm{mM})$, respectively, with trans-cinnamate being undetected. In contrast, 2phenylethanol was produced to a final titer of $98 \pm 3 \mathrm{mg} / \mathrm{L}$ $(0.80 \pm 0.02 \mathrm{mM})$ by BY4741 $\triangle F D C 1-\mathrm{PAL}$ with styrene being undetected throughout. In addition, in this case the extracellular accumulation of trans-cinnamate was also observed, reaching final concentration of $26 \pm 3 \mathrm{mg} / \mathrm{L}(0.18 \pm$ $0.02 \mathrm{mM}$ ) by $24 \mathrm{~h}$. Only trace levels of 2 -phenylacetate were observed throughout.

These results demonstrate several key points. First, styrene can be produced from phenylalanine by $S$. cerevisiae via the heterologous expression of PAL2 and native expression of FDC1. Second, both styrene and trans-cinnamate are naturally excreted from $S$. cerevisiae, at least to a certain degree. Third, since trans-cinnamate did not accumulate in BY4741-PAL cultures, this implies that the net activity afforded by native FDC1 expression was sufficiently high so as to preclude the creation of a flux bottleneck at the final pathway step (at least with respect to the specific PAL2 expression level examined). And, lastly, that under the conditions examined, synthesis of byproduct 2-phenylethanol significantly competes with the styrene pathway for precursor availability, even when

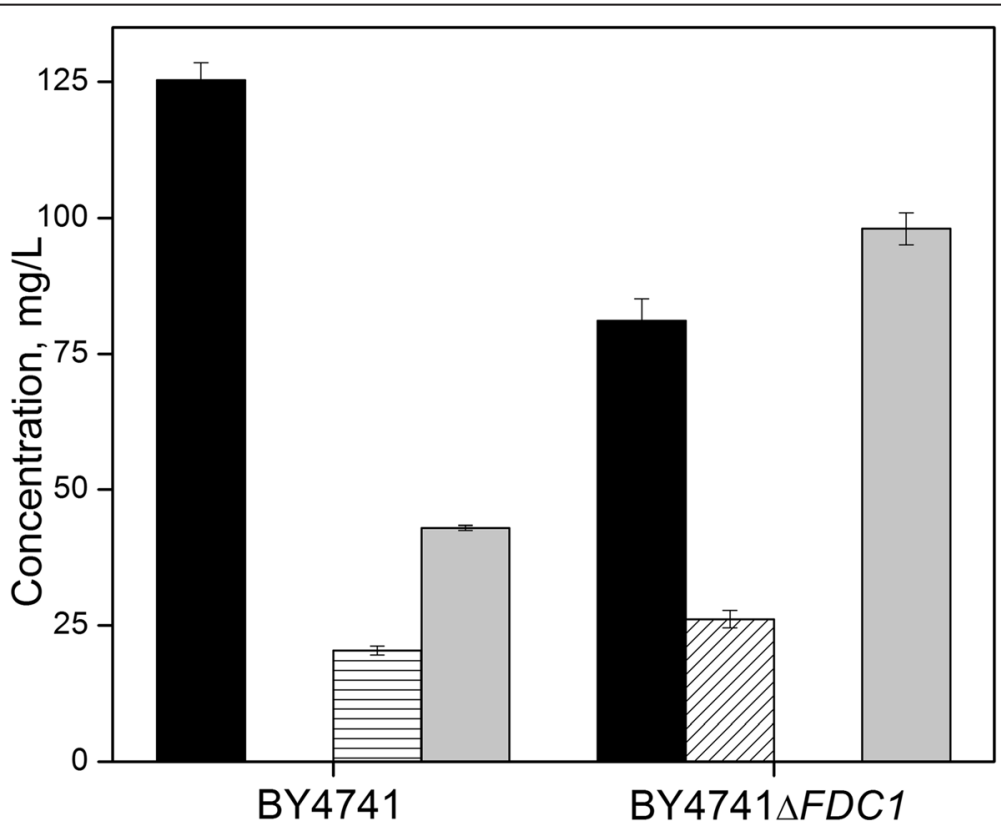

Figure 4 Assessing the trans-membrane export of trans-cinnamate. Depletion of exogenous phenylalanine (black; initially 200 mg/L) by growing cultures of wild-type S. cerevisiae BY4741-PAL and BY4741 $F$ FC1-PAL and the resultant production of trans-cinnamate (diagonal), styrene (horizontal), and 2-phenylethanol (gray) after $24 \mathrm{~h}$. Error bars reported at one standard deviation from triplicate experiments. 
PAL2 is constitutively expressed on a high copy number plasmid.

\section{Styrene production from glucose}

Based on the above findings, a series of strains were next constructed and evaluated with respect to their styrene production potential from glucose, the results of which are compared in Figure 5. Although only as much as $\sim 5 \mathrm{mg} / \mathrm{L}(0.05 \mathrm{mM})$ styrene was detected in BY4741PAL cultures, 22A75D-PAL accumulated up to $18 \pm$ $2 \mathrm{mg} / \mathrm{L}(0.17 \pm 0.02 \mathrm{mM})$ styrene in $48 \mathrm{~h}$. In the latter culture, however, more significant accumulation of byproduct 2-phenylethanol was also observed, reaching up to $54 \pm 5 \mathrm{mg} / \mathrm{L}(0.44 \pm 0.04 \mathrm{mM}$; again, 2-phenylacetate did not accumulate above trace levels). As this again suggested that the native Ehrlich pathway was competitively inhibiting the styrene pathway with respect to precursor availability, the effect of deleting ARO10 - which converts phenylpyruvate to phenylacetaldehyde $[32,43]$ - on styrene production was explored. As seen in Figure 5, deletion of ARO10 to preserve phenylpyruvate availability improved styrene production by 22A75D10-PAL by $~ 28 \%$, reaching up to $23 \pm 2 \mathrm{mg} / \mathrm{L}(0.22 \pm 0.02 \mathrm{mM})$. Lastly, to further improve styrene production, a feedback resistant mutant of $A R O 4$ - namely $A R O 4^{K 229 L}$ - was introduced into 22A75D10-PAL. Although ARO4 encodes a tyrosinesensitive DAHP synthase, in previous works $A R O 4^{K 229 L}$ over-expression in S. cerevisiae was shown to increase flux through the shikimic acid pathway by as much as 4.5 -fold $[16,24]$. Here, expressing ARO4 ${ }^{K 229 L}, 22 \mathrm{~A} 75 \mathrm{D} 104-\mathrm{PAL}$ displayed an additional $25 \%$ increase in styrene titer, reaching up to $29 \pm 2 \mathrm{mg} / \mathrm{L}(0.28 \pm 0.02 \mathrm{mM})$ at a glucose yield of about $1.44 \pm 0.11 \mathrm{mg} / \mathrm{g}(0.0025 \pm 0.0002 \mathrm{~mol} / \mathrm{mol}$; or just $0.6 \%$ of theoretical). Meanwhile, trans-cinnamate was not detected in the culture media of any styrene producing strain at any time (data not shown).

As a volatile product, it was also noted that styrene significantly accumulated within the headspace of each sealed flask. We have previously confirmed that said vapor-liquid partitioning behaves according to Henry's Law, with a dimensionless Henry's Law coefficient of 0.113 at $32^{\circ} \mathrm{C}[10]$. For the conditions examined here, this meant that an additional $\sim 45 \%$ styrene was produced and accumulated in each case. With this in mind, the maximum volumetric styrene production achieved here would be more accurately represented as $42 \pm$ $3 \mathrm{mg} / \mathrm{L}(0.40 \pm 0.03 \mathrm{mM})$ with a glucose yield of $2.09 \pm$ $0.16 \mathrm{mg} / \mathrm{g}(0.0036 \pm 0.0003 \mathrm{~mol} / \mathrm{mol} ; 0.9 \%$ of theoretical). The volatile nature of styrene may also prove useful as a product recovery strategy in the future [44].

Even at this adjusted output, however, achievable styrene production remains only $18 \%$ of the net production of 2-phenylethanol and 2-phenylacetate demonstrated by $22 \mathrm{~A} 75 \mathrm{D}(2.17 \pm 0.22 \mathrm{mM})$ and is $65 \%$ of the net production of styrene and 2-phenylethanol by 22A75D-PAL $(0.61 \pm 0.02 \mathrm{mM})$. This suggests that multiple limiting factors may have arisen during strain construction. Although it was not anticipated to be problematic at such low aqueous titers, to ensure that end-product inhibition was not the central productivity-limiting factor, a

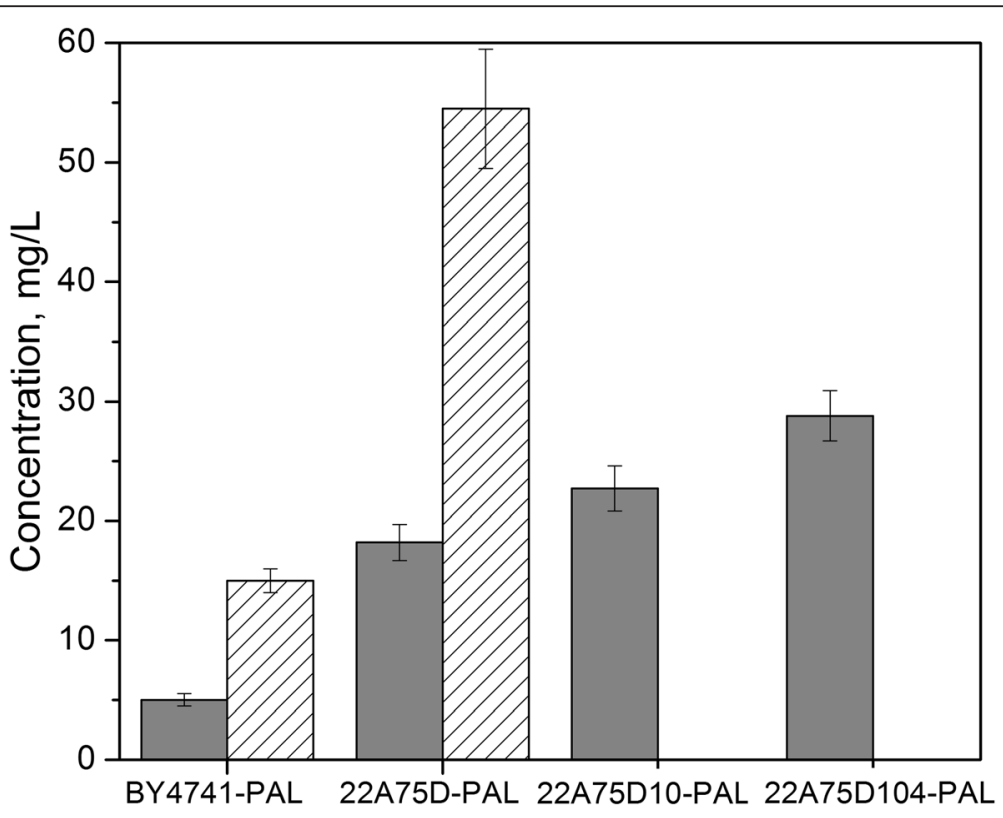

Figure 5 Styrene biosynthesis from glucose by engineered S. cerevisiae strains. Styrene (gray) and 2-phenylethanol (lined) production by strains BY4741-PAL, 22A75D-PAL, 22A75D10-PAL, and 22A75D104-PAL after $48 \mathrm{~h}$ in SD-Leu minimal media in sealed shake flask cultures. Error bars reported at one standard deviation from triplicate experiments. 
cursory evaluation of styrene toxicity was performed. $S$. cerevisiae growth and viability was found to be only minimally disrupted in the presence of styrene at up to at least $200 \mathrm{mg} / \mathrm{L}$ (1.92 mM; data not shown), suggesting that styrene toxicity was not a critical barrier at this point. Over-expression of PAL2 appears to have had the greatest negative impact, reducing net aromatic production by $72 \%$. This was likely due to the metabolic burden imposed by its expression from a high-copy plasmid. While decreasing PAL2 expression will likely reduce burden, as trans-cinnamate was never detected in styrene producing cultures, this already points to the fact that PAL2 activity was rate-limiting in the styrene pathway. Thus, future and careful optimization of PAL2 expression and/or the identification of other PAL homologs displaying greater inherent activity in $S$. cerevisiae will be key to achieving further improvements in styrene production.

Lastly, although seemingly low, it should be appreciated that level of styrene production demonstrated here agrees well with that of prior reports by others whom have engineered $S$. cerevisiae to produce aromatic chemicals (for which Curran et al. previously provided a comprehensive examination). Notable examples include $p$-hydroxycinnamate [45], $p$-aminobenzoic acid [46], $p$ hydroxybenzoic acid [46], and vanillin [20], which have been produced to maximal titers of up to 33.3, 34.3, 89.8 , and $45 \mathrm{mg} / \mathrm{L}$, respectively, at yields of $1.7,2.3,6.0$, and $2.3 \mathrm{mg} / \mathrm{g}$. Meanwhile, when compared with the baseline for styrene production established using an $E$. coli platform [10], the achievable titers and yields demonstrated with S. cerevisiae currently lag by about 9- and 21-fold, respectively. To achieve higher styrene titers with $S$. cerevisiae, further de-regulation of metabolite flux through its phenylalanine biosynthesis pathway will ultimately be required.

\section{Conclusions}

By coupling the classical approach of metabolic evolution with systematic strain and pathway engineering, styrene bioproduction directly from glucose by $S$. cerevisiae has been demonstrated for the first time. While providing an important demonstration of concept, future strain engineering efforts will be required to ultimately achieve economical production levels.

\section{Materials and methods}

\section{Strains and media}

All strains and plasmids used in this study are listed in Table 2. Custom oligonucleotide primers were synthesized by Integrated DNA Technologies (Coralville, IA) and are provided in Additional file 1: Table S1. All S. cerevisiae strains were purchased from Thermo Scientific (Waltham, MA). Yeast plasmids used were derived from the Gateway vector collection and purchased from AddGene (Cambridge, MA). Genomic DNA was prepared from $S$. cerevisiae whole cells using the ZR Fungal/Bacterial DNA MiniPrep (Zymo Research, Irvine, CA) according to vendor protocols. E. coli strain NEB10 $\beta$ (New England Biolabs, Ipswich, MA) was used for routine cloning and plasmid propagation, except for pDONR221 which was propagated in One Shot $c c d B$ Survival 2 T1 E. coli (Life Technologies, Grand Island, $\mathrm{NY}$ ). E. coli strains were routinely cultured at $37^{\circ} \mathrm{C}$ in Luria-Bertani (LB) broth supplemented with appropriate antibiotics, as necessary. Yeast strains were routinely cultured at $32^{\circ} \mathrm{C}$ in Yeast Extract Peptone Dextrose (YPD) medium, yeast synthetic dextrose (SD) medium, or yeast synthetic minimal (SD-Leu) medium. YPD medium was composed of $10 \mathrm{~g} / \mathrm{L}$ yeast extract, $20 \mathrm{~g} / \mathrm{L}$ peptone, and $20 \mathrm{~g} / \mathrm{L}$ glucose. SD medium was composed of $6.7 \mathrm{~g} / \mathrm{L}$ yeast nitrogen base, $20 \mathrm{~g} / \mathrm{L}$ glucose, and $20 \mathrm{mg} / \mathrm{L}$ of each uracil, histidine, leucine, and methionine. SD-Leu medium was composed of $6.7 \mathrm{~g} / \mathrm{L}$ yeast nitrogen base, $20 \mathrm{~g} / \mathrm{L}$ glucose, $20 \mathrm{mg} / \mathrm{L}$ each uracil, histidine, and methionine.

\section{Evolution of phenylalanine over-producing mutants}

Evolution of a phenylalanine over-producing phenotype in $S$. cerevisiae was achieved through random mutagenesis and high-throughput selection. The phenylalanine anti-metabolite $m$-fluoro-DL-phenylalanine provided selective pressure. S. cerevisiae BY4741 was first treated with ethylmethanesulphonate (EMS) according to standard protocols [47] before then being plated on minimal media supplemented with $m$-fluoro-DL-phenylalanine. In the first round of mutagenesis, selection occurred on SD media plates supplemented with either 18 or $22 \mathrm{mg} /$ $\mathrm{L} m$-fluoro-DL-phenylalanine. Note that the minimum inhibitory concentration of $m$-fluoro-DL-phenylalanine against BY4741 was initially determined as $\sim 15 \mathrm{mg} / \mathrm{L}$ (data not shown). Mutants isolated from the first round were then subjected to a second round of mutagenesis and selection, however, in this case using 25, 50, or $75 \mathrm{mg} / \mathrm{L} \mathrm{m}$-fluoro-DL-phenylalanine. All isolated $\mathrm{mu}$ tants were then cultured in SD media at $32^{\circ} \mathrm{C}$ for $48 \mathrm{~h}$ and periodically sampled and analyzed by high performance liquid chromatography (HPLC; as described below) to assess their comparative abilities with respect to producing 2-phenylethanol and 2-phenylacetate. Note that 2-phenylethanol and phenylacetate, which are endogenously produced from phenylpyruvate via ARO10 and either a native alcohol dehydrogenase (i.e., ADH1-6, and others putative dehydrogenases) or phenylacetaldehyde dehydrogenase (i.e., ALD1-6), respectively, were collectively used as surrogates to indicate enhanced flux through the phenylalanine biosynthesis pathway due to the fact that phenylalanine is not naturally exported by 
Table 2 List of strains and plasmids engineered and/or used in this study

\begin{tabular}{|c|c|c|}
\hline Strain & Genotype & Source \\
\hline \multicolumn{3}{|l|}{ E. coli } \\
\hline NEB10ß & $\begin{array}{l}\text { araD139 } \Delta(\text { ara,leu)7697 fhuA lacX74 galK16 galE15 mcrA f80d(lacZMM15)recA1 relA1 endA1 nupG rpsL rph } \\
\text { spoT1A(mrr-hsdRMS-mcrBC) }\end{array}$ & $\begin{array}{l}\text { New England } \\
\text { Biolabs }\end{array}$ \\
\hline $\begin{array}{l}\text { One Shot } c c d B \text { Survival } \\
2 \mathrm{~T} 1\end{array}$ & 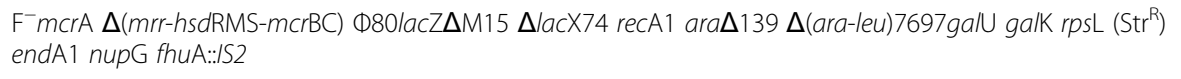 & $\begin{array}{l}\text { Life } \\
\text { Technologies }\end{array}$ \\
\hline \multicolumn{3}{|l|}{ S. cerevisiae } \\
\hline BY4741 & MATa his $3 \Delta 0$ leu2 $\Delta 0$ met $15 \Delta 0$ ura3 $\Delta 0$ & Thermo Scientific \\
\hline $\begin{array}{l}\text { BY4741 } \triangle F D C 1 \\
\text { (YDR539W) }\end{array}$ & MATa his $3 \Delta 0$ leu $2 \Delta 0$ met $15 \Delta 0$ ura $3 \Delta 0$ fdci $\Delta$ & Thermo Scientific \\
\hline $22 \mathrm{~A}$ & phenylalanine overproducer evolved from BY4741 & This Study \\
\hline 22A75D & phenylalanine overproducer evolved from 22A & This Study \\
\hline BY4741-PAL & MATa his $3 \Delta 0$ leu $2 \Delta 0$ met15 $\Delta 0$ ura3 $\Delta 0$ p425GPDPAL & This Study \\
\hline BY4741 $\triangle$ FDC1-PAL & MATa his $3 \Delta 0$ leu2 $\Delta 0$ met $15 \Delta 0$ ura3 $\Delta 0$ fdc $1 \Delta$ p425GPDPAL & This Study \\
\hline 22A75D-PAL & 22A75D harboring p425GPDPAL & This Study \\
\hline 22A75D10-PAL & 22A75D aro $10 \Delta$ harboring p425GPDPAL & This Study \\
\hline 22A75D104-PAL & 22A75D aro $10 \Delta:: a r o 4^{K 229 L}$ harboring p425GPDPAL & This Study \\
\hline \multicolumn{3}{|l|}{ Plasmids } \\
\hline pTrc99A & $P_{\text {tra }}$ pBR322 ori, lac $^{9}, A$ Amp $^{R}$ & Prather Lab, MIT \\
\hline pFA6-KanMX & KanR2, pBR322 ori, $A m p^{R}$ & AddGene \\
\hline pDONR221 & attP1-ccdB-Cm ${ }^{R}$-attP2 cassette, pUC ori, $K^{2}{ }^{R}$ & $\begin{array}{l}\text { Life } \\
\text { Technologies }\end{array}$ \\
\hline pDONR-PAL & PAL2 from A. thaliana inserted into pDONR221 & This study \\
\hline p425GPD & $P_{G P D}$ attR1-ccdB-Cm ${ }^{R}$-attR2 cassette, pBR322 ori, LEU2, Amp $p^{R}$ & AddGene \\
\hline p425GPDPAL & PAL2 from A. thaliana inserted into p425GPD & This study \\
\hline pACYCDuet-1 & $P_{\text {T7lac }}$ p15A ori, $\left.l a c\right|^{9}, \mathrm{Cm}^{R}$ & Novagen \\
\hline $\begin{array}{l}\text { PACYC-ARO4 }{ }^{\text {K229L }} \\
\text { KanMX }\end{array}$ & pACYCDuet-1 with the integration cassette aro $^{K 229 L}$-KanMX & This Study \\
\hline pUN15-PAL2 & Clone U12256 containing AT3G53260 (PAL2) from A. thaliana & $\mathrm{ABRC}$ \\
\hline
\end{tabular}

yeast to the extracellular media [24]. Several key pathway and regulatory genes (specifically, ARO3, ARO4, ARO7, ARO8, GCN4, and PHA2) were sequenced in each of BY4741 (wild type control) and top evolved mutants. In all cases, both the entire coding regions as well as 500 bp upstream of the start codon were sequenced.

\section{Transcriptional analysis of phenylalanine over-producing mutants}

Relative transcription levels of each of $A R O 1, A R O 2$, ARO3, ARO4, ARO7, ARO8, ARO9, and PHA2 were quantified at mid-log phase in each of BY4741 and the evolved mutants 22A and 22A75D. Approximately $1.5 \times$ $10^{8}$ cells of each of strain were collected by centrifugation at $17,000 \times g$ for $1 \mathrm{~min}$. The supernatant was discarded and RNA was extracted from the cell pellet using the YeaStar RNA Extraction Kit (Zymo Research, Irvine, CA). cDNA was synthesized using the SuperScript VILO cDNA Synthesis Kit (Life Technologies) whereas RT-qPCR was performed using SYBR Green (Life Technologies) based quantitative PCR according to manufacturer's protocols. Custom oligonucleotide primers for RT-qPCR experiments, including those for the reference housekeeping gene $26 \mathrm{~S}$ [48], were designed and synthesized, the sequences of which are provided in Additional file 1: Table S2. RT-qPCR was performed on an Applied Biosystems StepOne RealTime PCR (Applied Biosystems) using a $60^{\circ} \mathrm{C}$ annealing temperature. Data analysis was performed using StepOne software with relative transcriptional levels calculated via the $2^{-\Delta \Delta \mathrm{Ct}}$ method [49].

\section{Investigating native induction and activity of FDC1}

A seed culture of $S$. cerevisiae BY4741 were prepared in $5 \mathrm{~mL}$ YPD broth at $32^{\circ} \mathrm{C}$ while shaking at $250 \mathrm{rpm}$ overnight. The same seed $(1 \mathrm{~mL})$ was then used to inoculate a series of sealed shake flasks $(250 \mathrm{~mL})$ each containing $50 \mathrm{ml} \mathrm{SD}$ media. Cultures were grown until reaching an $\mathrm{OD}_{600}$ of $\sim 0.6$, at which point either trans-cinnamate, ferulate, $p$-coumarate, or phenylalanine were added to a final concentration of $0.2 \mathrm{mM}$. All cultures were 
incubated for an additional $12 \mathrm{~h}$ after which an equal number of cells $\left(\sim 7.4 \times 10^{7}\right)$ were collected by centrifugation at 2,800 $\mathrm{x} g$ for $4 \mathrm{~min}$. The cell pellet was lysed using Zymolyase (Zymo Research) before the sample was then centrifuged at $11,000 \times g$ for 2 min to separate and collect the supernatant. Each sample was then assayed for its ability to catalyze the decarboxylation of trans-cinnamate to styrene by adding $5 \mu \mathrm{L}$ of crude cell lysate to a $1 \mathrm{~mL}$ solution of $50 \mathrm{mM}$ Tris- $\mathrm{HCl}$ buffer (pH 7.5) initially containing $250 \mathrm{mM}$ trans-cinnamate. All samples were incubated at room temperature with the subsequent accumulation of styrene in the reaction mixture then followed at $247 \mathrm{~nm}$ for a total of $5 \mathrm{~min}$ at $20 \mathrm{~s}$ intervals using a UV/Vis spectrophotometer. A molar extinction coefficient of $10,000 \mathrm{M}^{-1} \mathrm{~cm}^{-1}$ [50] and a $1 \mathrm{~cm}$ path length were used to establish activity in terms of $\mathrm{mU} \mathrm{mg}^{-1}$ total protein. Total protein content in crude lysates was determined by Bradford Assay using bovine serum albumin (BSA) as an external standard.

\section{Cloning of PAL2 from A. thaliana}

The PAL2 encoding gene from A. thaliana was derived from a cDNA library plasmid containing the specific loci of interest (AT3G53260) and obtained from the Arabidopsis Biological Research Center (ABRC; Ohio State University, Columbus, OH). PAL2 was PCR amplified using Phusion DNA Polymerase (Finnzymes, Espoo, Finland) and custom oligonucleotide primers (Additional file 1: Table S1). Using Gateway Cloning Technology [19], amplified linear DNA fragments flanked with attB sequences were purified using the Zyppy Clean and Concentrator kit (Zymo Research). The BP reaction between the DNA fragment and pDONR221 (Life Technologies) was performed using Gateway BP Clonase II Enzyme Mix (Life Technologies) following manufacturer's protocols. Transformations were performed using chemically competent NEB10 $\beta$ with transformants being selected by plating overnight on LB solid agar containing $34 \mathrm{mg} / \mathrm{L}$ kanamycin. The resultant donor plasmid, pDONR-PAL, was mixed with the desired destination plasmid, p425GPD, using the Gateway LR Clonase II Enzyme Mix (Life Technologies), with transformation and selection as previously performed. As listed in Table 2, this approach resulted in construction of p425GPDPAL, a high copy $(2 \mu)$ plasmid for the constitutive expression of PAL2 in S. cerevisiae.

\section{Assaying the extracellular transport potential of trans- cinnamate}

S. cerevisiae BY4741 and the FDC1 deletion mutant BY4741 $\triangle F D C 1$ were each transformed with plasmid p425GPDPAL, resulting in strains BY4741-PAL and BY4741 $\triangle F D C 1-\mathrm{PAL}$, respectively. All yeast transformations were performed by lithium acetate method [51]. Both strains were then cultured in SD-Leu media. Upon reaching $\mathrm{OD}_{600}$ of $\sim 0.6,200 \mathrm{mg} / \mathrm{L}$ phenylalanine was then added to each culture, after which the extracellular accumulation of each of trans-cinnamate, styrene, and 2-phenylethanol were then periodically monitored via HPLC for a total period of $24 \mathrm{~h}$.

\section{Chromosomal disruption of ARO10 and integration of $\mathrm{ARO}^{\mathrm{K} 229 \mathrm{~L}}$}

Targeted chromosomal disruption of ARO10 in strain 22A75D was performed via homologous recombination. Gene disruption cassettes were generated via PCR to contain 40 base pairs of homology on both sides of the targeted integration site (i.e., ARO10), in addition to the KanMX selectable marker (as obtained from pFA6KanMX4). Following transformation, colonies were selected on YPD solid agar plates containing $200 \mathrm{mg} /$ L G418. Clones successfully carrying the ARO10 disruption cassette were further confirmed by colony PCR. This resulted in the strain 22A75D10. In addition, a copy of the feedback resistant mutant $A R O 4^{K 229 L}$, whose expression was driven by the native $A R O 4$ promoter, was likewise integrated into 22A75D at the ARO10 locus, thereby also and simultaneously resulting in the chromosomal disruption of ARO10. In this case, however, the gene disruption cassette was first constructed in the $E$. coli expression vector pACYCDuet-1. The ARO4 point mutation (K229L) was generated via overlap extension using primers as listed in Additional file 1: Table S1. The resultant fragment containing ARO4 ${ }^{K 229 L}$ was inserted between the BamHI and EcoRI sites of pACYCDuet-1, before the desired mutation was then confirmed by sequencing. Subsequently, the KanMX selectable marker with its promoter was PCR amplified from pFA6-KanMX4 before then being inserted downstream of $A R O 4^{K 229 L}$ to generate the plasmid pACYCARO4 ${ }^{\text {K229L }}$-KanMX. The entire cassette was then PCR amplified using primers whose overhangs each contained 40 base pairs of homology to ARO10. The resultant fragment was then transformed into 22A74D, followed by selection on YPD solid agar plates containing $200 \mathrm{mg} /$ L G418. Successful clones were further confirmed by colony PCR, resulting in strain 22A75D104.

\section{Styrene production from glucose by $\mathrm{S}$. cerevisiae}

S. cerevisiae BY4741, 22A75D, 22A75D10, and 22A75D104 were each individually transformed with the plasmid p425GPDPAL. The resultant strains (BY4741-PAL, 22A75DPAL, 22A75D10-PAL, and 22A75D104-PAL, respectively) were then each grown in $5 \mathrm{~mL}$ SD-Leu broth for $12 \mathrm{~h}$ at $32^{\circ} \mathrm{C}$ while shaking at $250 \mathrm{rpm}$ to prepare seed cultures. Each seed $(1 \mathrm{~mL})$ was then used to inoculate $50 \mathrm{~mL}$ SD-Leu media in a $250 \mathrm{ml}$ shake flask sealed with a glass cap. A closed system with large headspace was used to avoid volatile product (i.e., styrene) losses while also precluding the 
exhaustion of oxygen. Culturing continued for $48 \mathrm{~h}$ with periodic sampling performed to determine cell growth, residual substrate levels, and metabolite production.

\section{HPLC analysis}

Substrate and metabolite concentrations were determined via HPLC analysis. Samples were prepared by first removing $1 \mathrm{~mL}$ of culture from shake flasks and pelleting the cells at $11,000 \times \mathrm{g}$ for $2 \mathrm{~min}$. The supernatant $(0.75 \mathrm{~mL})$ was then transferred to a glass HPLC vial which was then sealed with a Teflon-lined screw cap. Separation and analysis was performed using a Hewlett Packard 1100 series HPLC system (Palo Alto, CA) equipped with an auto sampler, diode array (UV/Vis) detector, and reverse-phase Hypersil Gold SBC18 column (4.6 mm x $150 \mathrm{~mm}$; Thermo Fisher, USA), according to previously described methods [10]. The eluent was monitored at $215 \mathrm{~nm}$ for phenylalanine, 2-phenylethanol, and 2-phenylacetate, and at $258 \mathrm{~nm}$ for trans-cinnamate and styrene. Using the same HPLC system, glucose analysis was performed using an RID detector and an anion exchange column (Aminex HPX-87H; BioRAD, Hercules, CA). The column was eluted with $0.005 \mathrm{M} \mathrm{H}_{2} \mathrm{SO}_{4}$ at a constant flow rate of $0.8 \mathrm{ml} / \mathrm{min}$.

\section{Additional file}

Addtional file 1: Further details of relevance to this study.

\section{Competing interests}

The authors declare that they have no competing interests.

\section{Authors' contributions}

RM, BT, SP, and DN designed and performed the experiments, analyzed and interpreted the data. RM and DN conceived and supervised the research, as well as wrote the manuscript. All authors read and approved the final manuscript.

\section{Acknowledgements}

We thank Dr. Bo Wang (ASU) for his technical assistance with RT-qPCR experiments.

Received: 18 July 2014 Accepted: 14 August 2014

Published: 21 August 2014

\section{References}

1. Wu C, Koylinski T, Bozik J: Preparation of styrene from ethylbenzene US Patent 4,255,599. : 1981

2. Mimura N, Saito M: Dehydrogenation of ethylbenzene to styrene over $\mathrm{Fe}_{2} \mathrm{O}_{3} / \mathrm{Al}_{2} \mathrm{O}_{3}$ catalysts in the presence of carbon dioxide. Catal Today 2000, 55:173-178.

3. James DH, Castor WM: Styrene. In Ullmann's Encyclopedia of Industrial Chemistry, Volume Volume 34. 7th edition. Edited by Arpe HJ, Biekert E, Davis HT, Gerhartz W. Weinheim: Wiley-VCH; 2011.

4. SRI: Styrene. Rockville: Access Intelligence LLC Inc; 2010.

5. United States Department of Energy, Office of Energy Efficiency and Renewable Energy, National Renewable Energy Laboratory: Steam system opportunity assessment for the pulp and paper, chemical manufacturing, and petroleum refining industries. 2002

6. Adkins J, Pugh S, McKenna R, Nielsen DR: Engineering microbial chemical factories to produce renewable "biomonomers". Front Microbiol 2012, 3:313.
7. Curran KA, Alper HS: Expanding the chemical palate of cells by combining systems biology and metabolic engineering. Metab Eng 2012 14:289-297

8. EB N, Winters P: Perspective on opportunities in industrial biotechnology in renewable chemicals. Biotechnol J 2012, 7:176-185.

9. Lee JW, Kim HU, Choi S, Yi J, Lee SY: Microbial production of building block chemicals and polymers. Curr Opin Biotechnol 2011, 22:758-767.

10. McKenna R, Nielsen DR: Styrene biosynthesis from glucose by engineered E. coli. Metab Eng 2011, 13:544-554

11. Mukai N, Masaki K, Fujii T, Kawamukai M, lefuji H: PAD1 and FDC1 are essential for the decarboxylation of phenylacrylic acids in Saccharomyces cerevisiae. J Biosci Bioeng 2010, 109:564-569.

12. Claypool J, Raman DR, Jarboe L, Nielsen DR: Technoeconomic evaluation of bio-based styrene production by engineered Escherichia coli. J Ind Microbiol Biotechnol 2014 DOl:10.1007/s10295-014-1469-5.

13. Lo TM, Teo WS, Ling H, Chen BB, Kang A, Chang MW: Microbial engineering strategies to improve cell viability for biochemical production. Biotechnol Adv 2013, 31:903-914.

14. Demain AL, Vaishnav P: Production of recombinant proteins by microbes and higher organisms. Biotechnol Adv 2009, 27:297-306.

15. Sudbery PE: The expression of recombinant proteins in yeasts. Curr Opin Biotechnol 1996, 7:517-524

16. Curran KA, Leavitt JM, Karim AS, Alper HS: Metabolic engineering of muconic acid production in Saccharomyces cerevisiae. Metab Eng 2013, 15:55-66.

17. Nevoigt E: Progress in metabolic engineering of Saccharomyces cerevisiae. Microbiol Molec Biol Rev 2008, 72:379-412.

18. Ostergaard S, Olsson L, Nielsen J: Metabolic engineering of Saccharomyces cerevisiae. Microbiol Molec Biol Rev 2000, 64:34-50.

19. Alberti S, Gitler AD, Lindquist S: A suite of Gateway ${ }^{\circledast}$ cloning vectors for high-throughput genetic analysis in Saccharomyces cerevisiae. Yeast 2007, 24:913-919.

20. Hansen EH, Moller BL, Kock GR, Bunner CM, Kristensen C, Jensen OR, Okkels FT, Olsen CE, Motawia MS, Hansen J: De novo biosynthesis of vanillin in fission yeast (Schizosaccharomyces pombe) and baker's yeast (Saccharomyces cerevisiae). Appl Environ Microbiol 2009, 75:2765-2774.

21. Jiang $H$, Wood KV, Morgan JA: Metabolic engineering of the phenylpropanoid pathway in Saccharomyces cerevisiae. Appl Environ Microbiol 2005, 71:2962-2969.

22. Stark D, Munch T, Sonnleitner B, Marison IW, von Stockar U: Extractive bioconversion of 2-phenylethanol from L-phenylalanine by Saccharomyces cerevisiae. Biotechnol Prog 2002, 18:514-523.

23. Koopman F, Beekwilder J, Crimi B, van Houwelingen A, Hall RD, Bosch D, van Maris AJA, Pronk JT, Daran JM: De novo production of the flavonoid naringenin in engineered Saccharomyces cerevisiae. Microb Cell Fact 2012, 11:155-169

24. Luttik MAH, Vuralhan Z, Suir E, Braus GH, Pronk JT, Daran JM: Alleviation of feedback inhibition in Saccharomyces cerevisiae aromatic amino acid biosynthesis: Quantification of metabolic impact. Metab Eng 2008, 10:141-153

25. Nijkamp K, van Luijk N, de Bont JA, Wery J: The solvent-tolerant Pseudomonas putida S12 as host for the production of cinnamic acid from glucose. Appl Microbiol Biotechnol 2005, 69:170-177.

26. Nijkamp K, Westerhof RG, Ballerstedt H, de Bont JA, Wery J: Optimization of the solvent-tolerant Pseudomonas putida S12 as host for the production of p-coumarate from glucose. Appl Microbiol Biotechnol 2007, 74:617-624.

27. Verhoef S, Wierckx N, Westerhof RG, de Winde JH, Ruijssenaars HJ: Bioproduction of $\mathrm{p}$-hydroxystyrene from glucose by the solvent-tolerant bacterium Pseudomonas putida S12 in a two-phase water-decanol fermentation. Appl Environ Microbiol 2009, 75:931-936.

28. Wierckx NJ, Ballerstedt $H$, de Bont JA, Wery J: Engineering of solventtolerant Pseudomonas putida S12 for bioproduction of phenol from glucose. Appl Environ Microbiol 2005, 71:8221-8227.

29. Drake RH: The biochemistry of mutagenesis. Annu Rev Microbiol 1976 45:11-37.

30. Wloch DM, Szafraniec K, Borts RH, Korona R: Direct estimate of the mutation rate and the distribution of fitness effects in the yeast Saccharomyces cerevisiae. Genetics 2001, 159:441-452.

31. Vuralhan Z, Morais MA, Tai SL, Piper MD, Pronk JT: Identification and characterization of phenylpyruvate decarboxylase genes in Saccharomyces cerevisiae. Appl Environ Microbiol 2003, 69:4534-4541. 
32. Hazelwood LA, Daran JM, van Maris AJ, Pronk JT, Dickinson JR: The Ehrlich pathway for fusel alcohol production: a century of research on Saccharomyces cerevisiae metabolism. Appl Environ Microbiol 2008, 74:2259-2266.

33. Caspi R, Foerster $H$, Fulcher CA, Kaipa P, Krummenacker M, Latendresse M, Paley S, Rhee SY, Shearer AG, Tissier C, Walk TC, Zhang P, Karp PD, Caspi R, Foerster H, Fulcher CA, Hopkinson R, Ingraham J, Kaipa P, Krummenacker M, Paley S, Pick J, Rhee SY, Tisse C, Zhang P, Karp PD: MetaCyc: a multiorganism database of metabolic pathways and enzymes. Nucleic Acids Res 2006, 34:D511-D516.

34. Fukuda K, Watanabe M, Asano K, Ouchi K, Takasawa S: A mutated ARO4 gene for feedback-resistant DAHP synthase which causes both o-fluoro$\mathrm{dl}$-phenylalamine resistance and $\beta$-phenethyl-alcohol overproduction in Saccharomyces cerevisiae. Curr Genet 1991, 20:453-456.

35. Teshiba S, Furter R, Niederberger P, Braus G, Paravicini G, Hütter R: Cloning of the ARO3 gene of Saccharomyces cerevisiae and its regulation. Molec Gen Genet 1986, 205:353-357.

36. Schmidheini T, Sperisen P, Paravicini G, Hütter R, Braus G: A single point mutation results in a constitutively activated and feedback-resistant chorismate mutase of Saccharomyces cerevisiae. J Bacteriol 1989, 171:1245-1253.

37. Kradolfer P, Zeyer J, Miozzari G, Huetter R: Dominant regulatory mutants in chorismate mutase of Saccharomyces cerevisiae. FEMS Microbiol Lett 1977, 2:211-216.

38. Weber C, Bruckner C, Weinreb S, Lehr C, Essl C, Boles E: Biosynthesis of cis, cis-muconic acid and its aromatic precursors, catechol and protocatechuic acid, from renewable feedstocks by Saccharomyces cerevisiae. Appl Environ Microbiol 2012, 78:8421-8430.

39. Dickinson JR, Salgado LE, Hewlins MJ: The catabolism of amino acids to long chain and complex alcohols in Saccharomyces cerevisiae. J Biol Chem 2003, 278:8028-8034.

40. Iraqui I, Vissers S, Cartiaux M, Urrestarazu A: Characterisation of Saccharomyces cerevisiae $A R O 8$ and $A R O 9$ genes encoding aromatic aminotransferases I and II reveals a new aminotransferase subfamily. Molec Gen Genet 1998, 257:238-248.

41. Hinnebusch AG: Translational regulation of gcn4 and the general amino Acid control of yeast. Annu Rev Microbiol 2005, 59:407-450

42. Cherry JM, Hong EL, Amundsen C, Balakrishnan R, Binkley G, Chan ET, Christie KR, Costanzo MC, Dwight SS, Engel SR: Saccharomyces Genome Database: the genomics resource of budding yeast. Nucleic Acids Res 2012, 40:D700-D705.

43. Caspi R, Foerster $H$, Fulcher CA, Kaipa P, Krummenacker M, Latendresse M, Paley S, Rhee SY, Shearer AG, Tissier C, Walk TC, Zhang P, Karp PD: The MetaCyc database of metabolic pathways and enzymes and the BioCyc collection of pathway/genome databases. Nucleic Acids Res 2008, 36: D623-D631

44. McKenna R, Moya L, McDaniel M, Nielsen DR: Comparing in situ removal strategies for improving styrene bioproduction. Bioproc Biosys Eng 2014. DOl:10.1007/s00449-014-1255-9.

45. Vannelli T, Qi WW, Sweigard J, Gatenby AA, Sariaslani FS: Production of p-hydroxycinnamic acid from glucose in Saccharomyces cerevisiae and Escherichia coli by expression of heterologous genes from plants and fungi. Metab Eng 2007, 9:142-151.

46. Kromer JO, Nunez-Bernal D, Averesch NJH, Hampe J, Varela J, Varela C: Production of aromatics in Saccharomyces cerevisiae - a feasibility study. J Biotechnol 2013, 163:184-193.

47. Winston F: EMS and UV mutagenesis in yeast. In Current Protocols in Molecular Biology: John Wiley \& Sons, Inc.; 2001.

48. Martorell P, Querol A, Fernández-Espinar MT: Rapid identification and enumeration of Saccharomyces cerevisiae cells in wine by real-time PCR. Appl Environ Microbiol 2005, 71:6823-6830.
49. Livak KJ, Schmittgen TD: Analysis of relative gene expression data using real-time quantitative PCR and the 2(-Delta Delta C(T)) Method. Methods 2001, 25:402-408.

50. Murray MJ, Gallaway WS: Steric inhibition of resonance in 1-methyl-2isopropenylbenzene. J Am Chem Soc 1948, 70:3867-3868.

51. Gietz RD, Woods RA: Transformation of yeast by lithium acetate/singlestranded carrier DNA/polyethylene glycol method. Methods Enzymol 2002, 350:87-96.

doi:10.1186/s12934-014-0123-2

Cite this article as: McKenna et al:: Rational and combinatorial approaches to engineering styrene production by Saccharomyces cerevisiae. Microbial Cell Factories 2014 13:123.

\section{Submit your next manuscript to BioMed Central and take full advantage of:}

- Convenient online submission

- Thorough peer review

- No space constraints or color figure charges

- Immediate publication on acceptance

- Inclusion in PubMed, CAS, Scopus and Google Scholar

- Research which is freely available for redistribution

Submit your manuscript at www.biomedcentral.com/submit
C) BioMed Central 